\title{
BMJ Open Adherence to clinical practice guidelines (CPGs) for the treatment of cancers in Australia and the factors associated with adherence: a systematic review protocol
}

\author{
Mia Bierbaum (D) , ${ }^{1}$ Frances Rapport (D) , , Gaston Arnolda (D) , ${ }^{1}$ Yvonne Tran, ${ }^{1,2}$ \\ Bróna Nic Giolla Easpaig (D) , ${ }^{1}$ Kristiana Ludlow (D) , ${ }^{1,3}$ Jeffrey Braithwaite (D) ${ }^{1}$
}

To cite: Bierbaum M, Rapport F, Arnolda G, et al. Adherence to clinical practice guidelines (CPGs) for the treatment of cancers in Australia and the factors associated with adherence: a systematic review protocol. BMJ Open 2021;11:e050912. doi:10.1136/ bmjopen-2021-050912

- Prepublication history and additional supplemental material for this paper are available online. To view these files, please visit the journal online (http://dx.doi.org/10.1136/ bmjopen-2021-050912).

Received 03 March 2021 Accepted 07 September 2021

Check for updates

(c) Author(s) (or their employer(s)) 2021. Re-use permitted under CC BY-NC. No commercial re-use. See rights and permissions. Published by BMJ.

${ }^{1}$ Australian Institute of Health Innovation, Macquarie University, Sydney, New South Wales, Australia

${ }^{2}$ Macquarie University Hearing, Sydney, New South Wales, Australia

${ }^{3}$ The University of Queensland, School of Psychology, Saint Lucia, Queensland, Australia

Correspondence to

Mia Bierbaum;

mia.bierbaum@hdr.mq.edu.au

\section{ABSTRACT}

Introduction Clinical practice guidelines (CPGs) synthesise the latest evidence to support clinical and patient decision-making. CPG adherent care is associated with improved patient survival outcomes; however, adherence rates are low across some cancer streams in Australia. Greater understanding of specific barriers to cancer treatment CPG adherence is warranted to inform future implementation strategies.

This paper presents the protocol for a systematic review that aims to determine cancer treatment CPG adherence rates in Australia across a variety of common cancers, and to identify any factors associated with adherence to those CPGs, as well as any associations between CPG adherence and patient outcomes.

Methods and analysis Five databases will be searched, Ovid Medline, PsychInfo, Embase, Scopus and Web of Science, for eligible studies evaluating adherence rates to cancer treatment CPGs in Australia. A team of reviewers will screen the abstracts in pairs according to predetermined inclusion criteria and then review the full text of eligible studies. All included studies will be assessed for quality and risk of bias. Data will be extracted using a predefined data extraction template. The frequency or rate of adherence to CPGs, factors associated with adherence to those CPGs and any reported patient outcome rates (eg, relative risk ratios or 5-year survival rates) associated with adherence to CPGs will be described. If applicable, a pooled estimate of the rate of adherence will be calculated by conducting a randomeffects meta-analysis. The systematic review will adhere to the Preferred Reporting Items for Systematic Reviews and Meta-Analyses guidelines.

Ethics and dissemination Ethics approval will not be required, as this review will present anonymised data from other published studies. Results from this study will form part of a doctoral dissertation (MB), will be published in a journal, presented at conferences, and other academic presentations.

PROSPERO registration number CRD42020222962.

\section{INTRODUCTION}

Clinical practice guidelines (CPGs) synthesise the latest evidence to support clinical and patient decision-making, ${ }^{1}$ and are designed
Strengths and limitations of this study

- This systematic review protocol describes a planned review that will use an internationally recognised methodology for the collection, extraction and synthesis of data, enabling transparent presentation of methods and results to enable replication.

- The reporting of the systematic review will adhere to the Preferred Reporting Items for Systematic Reviews and Meta-Analyses guidelines.

- Abstracts and full texts will be reviewed by pairs of reviewers, with the lead reviewer reviewing all abstracts and full texts and inter-rater reliability scores will be calculated for both screening stages.

- The main limitation of the review will be the exclusion of studies published in languages other than English, and the exclusion of non-empirical research, including conference abstracts, editorials, opinion pieces and research which has not been peer reviewed.

- This study will also be limited to cancer treatmentspecific clinical practice guidelines (CPGs), excluding CPGs that relate to other elements of the cancer care journey such as pain management and psychosocial care.

to reduce clinical variation, through standardisation of clinical practice in line with best evidence, to ultimately enhance clinical outcomes. ${ }^{2}{ }^{3}$ There is growing evidence that CPG adherent cancer care is linked to improved patient survival rates. ${ }^{4-8}$ Despite this, adherence to cancer treatment CPGs in Australia is often reported to be low across a variety of cancer streams. ${ }^{46-14}$ Variation from CPG recommendations in some instances can be reasonably justified to account for individual patient characteristics and preferences. ${ }^{515}$ However, little is known about the factors that influence these decisions. ${ }^{16}$ Low uptake and adherence to cancer treatment CPGs may also reflect poor quality CPGs, or poor implementation of CPGs. Greater 
understanding of specific barriers to adherence to cancer treatment CPGs is warranted, to inform future implementation strategies.

There are a multitude of internal (attitudinal) and external (organisational and structural) barriers to cancer treatment CPG adherence. These include clinician concern that CPGs are biased or oversimplified, ${ }^{1517}$ a lack of clinician awareness of, or agreement with, CPG recommendations, ${ }^{15}{ }^{17}$ inertia $^{15} 17$ and concerns about the side effects of CPG recommended treatment. ${ }^{15}$ Similarly, patient concerns about side effects or discomfort related to therapy, and treatment access, have also been identified as barriers to CPG adherence. ${ }^{18}$ Collaboration within multidisciplinary teams during treatment decisionmaking has been found to influence adherence to CPG recommendations, ${ }^{19-21}$ as has patient access to services, ${ }^{4}$ the location of the treating hospital (rural, remote or metropolitan), ${ }^{22}$ as well as the patient receiving care at a different facility from the initial treatment centre. ${ }^{23}$ Additionally, out-of-date CPGs that do not represent the latest evidence, ${ }^{15}$ and a lack of resources or time to implement CPG recommendations ${ }^{1524}$ have been found to influence CPG adherence. Patient and clinician characteristics associated with CPG adherence include older patient age, ${ }^{4} 1114222325-28$ race, ${ }^{29}{ }^{30}$ gender, ${ }^{16}$ comorbid conditions, ${ }^{141625}$ patient private health insurance ${ }^{1423}$ and socioeconomic status, ${ }^{29} 31$ as well as clinician specialty ${ }^{22}$ and tumour-specific caseload. ${ }^{632}$

It is unknown whether these factors are associated with cancer treatment CPG adherence in Australia, and if there are similar patterns across cancer streams. Successful implementation of CPGs needs to be contextspecific. ${ }^{233}$ Therefore, the identification of factors specific to the Australian cancer treatment context is warranted in order to enable future CPG development, implementation and dissemination to be tailored according to identified facilitators and barriers of adherence within the country.

\section{Objectives}

The primary objective of the systematic review will be to:

- Determine the estimated rates of adherence to cancer treatment CPG recommendations in Australia.

The secondary objectives of the review will be to:

- Identify factors associated with adherence or nonadherence to cancer treatment CPGs in Australia, estimating the association between those factors and adherence.

- Identify if there are associations between cancer treatment CPG adherence and patient outcomes in Australia (where possible).

\section{METHODS AND ANALYSIS}

This systematic review will be conducted in accordance with the Preferred Reporting for Items for Systematic Reviews and Meta-Analyses (PRISMA) 2020 statement, ${ }^{34}$ and the protocol has been developed according to the PRISMA-P (protocol) checklist (online supplemental file 1). ${ }^{35}$ The systematic review database search began in March 2021, and it is anticipated that the review will be completed by March 2022. The PROSPERO registration will be updated if significant amendments are made to the protocol.

\section{Search strategy}

Five databases will be searched for eligible title/abstracts, including Ovid Medline, PsychInfo, Embase, Scopus and Web of Science (please see online supplemental file 2) for the detailed example of the search strategy applied in Embase). The abstracts identified will be collated in EndNote, with duplicates removed. The abstracts will then be reviewed for eligibility according to preestablished inclusion and exclusion criteria. Eligible studies will report the rate of adherence in Australia to cancer treatment CPGs. Examples of eligible study types include randomised studies, quasi-experimental designs and observational studies (eg, cohort studies, casecontrol studies) and mixed-methods studies that report quantitative data separately.

It is envisaged that some studies will include experimental interventions designed to influence adherence to CPG recommendations. Baseline data from experimental studies will be considered for meta-analysis along with data from observational studies; comparison of preintervention and postintervention data within experimental studies may provide information about barriers and facilitators.

\section{Inclusion criteria}

Studies must include empirical research, investigating the rate of receipt of cancer treatment CPG adherent care in Australia (including primary treatment, and neoadjuvant and adjuvant treatments), and factors associated with adherence to those CPGs. The review will include empirical research articles published in English, restricted to patient care within Australia. No date restrictions will be applied.

\section{Additional full text inclusion criteria}

The full text of each study must reference the relevant CPGs, and clearly describe how CPG adherence was measured.

\section{Exclusion criteria}

Conference abstracts, editorials and opinion pieces as well as purely qualitative research will be excluded. Studies that do not include patient care within Australia and studies focusing on diseases other than cancer will also be excluded. Studies that report data from both Australia and other countries will be excluded if the Australian data are not reported separately. This review will exclude studies referring to adherence to cancer prevention CPGs (eg, screening CPGs) and CPGs for cancer treatmentassociated side effects (eg, pain management CPGs, antiemetic prophylaxis CPGs and psychosocial care-focused CPGs, etc.). 


\section{Additional full-text exclusion criteria}

The full text of studies will be excluded if they do not identify the rate of receipt of CPG adherent care, and/or factors associated with adherence to those CPGs or fail to clearly indicate the CPG/s to which they refer.

\section{Screening}

1. A team of reviewers, experienced in conducting systematic reviews, will conduct the title abstract screen in pairs. To enhance consistency of inclusion and exclusion of abstracts, a $10 \%$ sample of title abstracts will be reviewed by the whole team and discussed. The remaining abstracts will be divided among the reviewers, with the lead author reviewing every abstract in conjunction with another reviewer. Each reviewer will be blinded to the lead author's decisions; disagreements will be resolved by reviewer pairs reaching a consensus. Inter-rater reliability will be assessed between the lead author and each of the additional reviewers. Title abstracts that meet inclusion criteria and do not clearly breach exclusion criteria will be selected for full-text review.

2. The full texts of the studies selected during the title abstract screen will be reviewed by the team in pairs. As in the abstract screening step, to enhance consistency of assessment of full texts, a 10\% sample of full texts will be independently reviewed by the team of reviewers blinded to other reviewer decisions. The remaining full texts will be divided among the team of reviewers, with the lead author reviewing every full text in conjunction with another reviewer. Disagreements will be resolved by reviewer pairs reaching a consensus. Inter-rater reliability between the lead author and each reviewer will be calculated for the full-text screening stage. All inter-rater reliability scores will be reported in the systematic review manuscript. Articles that meet the inclusion criteria and do not breach the exclusion criteria will be included in the review. The reason for exclusion of each reviewed article will be recorded on a data extraction template in Microsoft Excel. The reference lists of included papers will also be reviewed for potentially relevant articles not identified by database searches.

\section{Data extraction}

1. For all included articles, a data extraction template will be used to extract data. This template will be piloted by two reviewers on five full texts. Disagreements will be resolved through team consensus, and the tool will be revised if necessary. The template will extract the citation; location of study; study design; sample size; data source; age range, gender and race (if applicable) of participants; cancer stream(s); cancer stage(s); description of $\mathrm{CPG}(\mathrm{s})$ being assessed; measure of adherence; study intervention (if relevant); description of factors reported in studies that are associated with CPG adherence or non-adherence (eg, patient age, Charlson Comorbidity Index, Socioeconomic Status, geographic remoteness, Country of Birth, Eastern Cooperative Oncology Group performance status, Aboriginal and Torres Strait Islander status, clinician case load or hospital case load), including the strength of association of correlates with adherence/non-adherence; potential confounders (if available) and any available patient outcome data such as risk ratios, HRs, 5-year survival rates, patient satisfaction, etc. All cancer streams will be included in the review.

2. All included studies will be checked by a second reviewer to confirm accuracy of data extraction. Any disagreements will be resolved through team consensus.

\section{Risk of bias and strength of evidence assessment}

The quality appraisal and assessment of risk of bias for each included study will be conducted by two reviewers, experienced in conducting systematic reviews. The Joanna Briggs Institute checklists ${ }^{36}$ for each study type (eg, randomised control trials, cross-sectional studies, cohort studies or case-control studies) will be used to assess the quality and risk of bias of each study, noting that questions differ for assessment of each study type. For example, the checklist questions for assessment of cross-sectional studies include, ' 1 . Were the criteria for inclusion in the sample clearly defined? 2. Were the study subjects and the setting described in detail? 3. Was the exposure measured in a valid and reliable way? 4 . Were objective, standard criteria used for measurement of the condition? 5. Were confounding factors identified? 6 . Were strategies to deal with confounding factors stated? 7. Were the outcomes measured in a valid and reliable way? 8 . Was appropriate statistical analysis used?'. ${ }^{36}$ These data will be reported in the manuscript. Each risk of bias assessment will be checked by a second reviewer. Any disagreements will be resolved through team consensus.

\section{Strategy for data synthesis}

For each study, the frequency or rate of receipt of CPG adherent (or non-adherent) cancer treatment will be described. The factors associated with CPG adherent cancer treatment, including the strength of association, if available, will be described. The review will focus on variables that are significantly associated with CPG adherence in one or more studies. The categorisation of these variables will be guided by the WHO's five dimensions of adherence framework, which includes patient factors, socioeconomic factors, health condition factors, medical therapy factors, as well as factors related to the healthcare system and team. ${ }^{37}$

Where available, patient outcome rates (eg, HRs, 5-year survival rates) of patients in receipt of CPG adherent treatment, compared with those in receipt of CPG non-adherent treatment, will be described. A narrative synthesis of qualitative data regarding adherence rates or factors related to CPG adherence, from included mixedmethods studies, will be produced, if available. When two studies report adherence rates for a particular treatment, a pooled estimate of the rate of adherence (mean 
adherence) will be calculated by conducting a randomeffects meta-analysis, if appropriate. If non-adherence rates are reported, these will first be converted to adherence rates. Prior to pooling, forest plots, Q-tests or $\mathrm{I}^{2}$ tests will be used to assess heterogeneity. ${ }^{38}$ Funnel plots will be used to identify publication bias.

\section{Patient and public involvement}

Patients or the public were not involved in the design, or conduct, or reporting or dissemination plans of this research, and will not be involved in the research outlined by this protocol.

\section{ETHICS AND DISSEMINATION}

Ethics approval will not be required as this study will only present anonymised data from other published studies. On completion of the systematic review, results will be published in a peer-reviewed journal, and as part of a dissertation (MB). Results may also be presented at international and national conferences, as well as other academic presentations. To translate the research into action, the findings from this work will be distributed to guideline development bodies, clinical societies involved in cancer treatment in Australia and stakeholders involved in policy development and implementation in oncology.

\section{Twitter Mia Bierbaum @Mia_R_Bierbaum}

Contributors MB was responsible for the conception of the study plan, and preparation of the manuscript. JB, FR, GA, YT, BNGE and KL reviewed the study plan and manuscript and offered comments and edits.

Funding This work was supported by an Australian Government's Research Training Programme Scholarship associated with the Australian Institute of Health Innovation, Macquarie University, grant number ID:9100002 awarded to MB.

Competing interests None declared.

Patient consent for publication Not required.

Provenance and peer review Not commissioned; externally peer reviewed.

Supplemental material This content has been supplied by the author(s). It has not been vetted by BMJ Publishing Group Limited (BMJ) and may not have been peer-reviewed. Any opinions or recommendations discussed are solely those of the author(s) and are not endorsed by BMJ. BMJ disclaims all liability and responsibility arising from any reliance placed on the content. Where the content includes any translated material, BMJ does not warrant the accuracy and reliability of the translations (including but not limited to local regulations, clinical guidelines, terminology, drug names and drug dosages), and is not responsible for any error and/or omissions arising from translation and adaptation or otherwise.

Open access This is an open access article distributed in accordance with the Creative Commons Attribution Non Commercial (CC BY-NC 4.0) license, which permits others to distribute, remix, adapt, build upon this work non-commercially, and license their derivative works on different terms, provided the original work is properly cited, appropriate credit is given, any changes made indicated, and the use is non-commercial. See: http://creativecommons.org/licenses/by-nc/4.0/.

\section{ORCID iDs}

Mia Bierbaum http://orcid.org/0000-0002-7037-4708

Frances Rapport http://orcid.org/0000-0002-4428-2826

Gaston Arnolda http://orcid.org/0000-0003-4948-7633

Bróna Nic Giolla Easpaig http://orcid.org/0000-0001-6787-056X

Kristiana Ludlow http://orcid.org/0000-0001-7284-5625

Jeffrey Braithwaite http://orcid.org/0000-0003-0296-4957

\section{REFERENCES}

1 Grol R. Successes and failures in the implementation of evidencebased guidelines for clinical practice. Med Care 2001;39:I-46.

2 Harrison MB, Légaré F, Graham ID, et al. Adapting clinical practice guidelines to local context and assessing barriers to their use. CMAJ 2010;182:E78-84.

3 Bierbaum M, Braithwaite J, Arnolda G, et al. Clinicians' attitudes to oncology clinical practice guidelines and the barriers and facilitators to adherence: a mixed methods study protocol. BMJ Open 2020;10:e035448.

4 Adelson P, Fusco K, Karapetis C, et al. Use of guidelinerecommended adjuvant therapies and survival outcomes for people with colorectal cancer at tertiary referral hospitals in South Australia. J Eval Clin Pract 2018;24:135-44.

5 Balasubramanian SP, Murrow S, Holt S, et al. Audit of compliance to adjuvant chemotherapy and radiotherapy guidelines in breast cancer in a cancer network. Breast 2003;12:136-41.

6 Bristow RE, Chang J, Ziogas A, et al. Adherence to treatment guidelines for ovarian cancer as a measure of quality care. Obstet Gynecol 2013;121:1226-34.

7 Chiew K-L, Chong S, Duggan KJ, et al. Assessing guideline adherence and patient outcomes in cervical cancer. Asia Pac J Clin Oncol 2017;13:e373-80.

8 Rossi CR, Vecchiato A, Mastrangelo G, et al. Adherence to treatment guidelines for primary sarcomas affects patient survival: a side study of the European connective tissue cancer network (CONTICANET). Ann Oncol 2013;24:1685-91.

9 Ebner F, Hancke K, Blettner M, et al. Aggressive Intrinsic Subtypes in Breast Cancer: A Predictor of Guideline Adherence in Older Patients With Breast Cancer? Clin Breast Cancer 2015;15:e189-95.

10 Rayson D, Urquhart R, Cox M, et al. Adherence to clinical practice guidelines for adjuvant chemotherapy for colorectal cancer in a Canadian Province: a population-based analysis. J Oncol Pract 2012;8:253-9.

11 Duggan KJ, Descallar J, Vinod SK. Application of guideline recommended treatment in routine clinical practice: a populationbased study of stage I-IIIB non-small cell lung cancer. Clin Oncol 2016;28:639-47.

12 Merie R, Gabriel G, Shafiq J, et al. Radiotherapy underutilisation and its impact on local control and survival in New South Wales, Australia. Radiother Oncol 2019;141:41-7.

13 Landercasper J, Dietrich LL, Johnson JM. A breast center review of compliance with national comprehensive cancer network breast cancer guidelines. Am J Surg 2006;192:525-7.

14 Chagpar R, Xing Y, Chiang Y-J, et al. Adherence to stage-specific treatment guidelines for patients with colon cancer. J Clin Oncol 2012;30:972-9.

15 Bierbaum M, Rapport F, Arnolda G, et al. Clinicians' attitudes and perceived barriers and facilitators to cancer treatment clinical practice guideline adherence: a systematic review of qualitative and quantitative literature. Implementation Science 2020;15:1-24.

16 Dronkers EAC, Mes SW, Wieringa MH, et al. Noncompliance to guidelines in head and neck cancer treatment; associated factors for both patient and physician. BMC Cancer 2015;15:515.

17 Cabana MD, Rand CS, Powe NR, et al. Why don't physicians follow clinical practice guidelines? A framework for improvement. JAMA 1999;282:1458-65.

18 Puts MTE, Tapscott B, Fitch M, et al. A systematic review of factors influencing older adults' decision to accept or decline cancer treatment. Cancer Treat Rev 2015;41:197-215.

19 Brouwers MC, Makarski J, Garcia K, et al. A mixed methods approach to understand variation in lung cancer practice and the role of guidelines. Implement Sci 2014;9:36.

20 Fonteyne V, Rammant E, Ost P, et al. Evaluating the current place of radiotherapy as treatment option for patients with muscle invasive bladder cancer in Belgium. Clin Genitourin Cancer 2018;16:e1159-69.

21 Gattellari M, Ward J, Solomon M. Implementing guidelines about colorectal cancer: a national survey of target groups. ANZ J Surg 2001;71:147-53.

22 Young JM, Leong DC, Armstrong K, et al. Concordance with national guidelines for colorectal cancer care in New South Wales: a population-based patterns of care study. Med J Aust 2007;186:292-5.

23 Boland GM, Chang GJ, Haynes AB, et al. Association between adherence to national comprehensive cancer network treatment guidelines and improved survival in patients with colon cancer. Cancer 2013;119:1593-601.

24 Ploeg J, Davies B, Edwards N, et al. Factors influencing bestpractice guideline implementation: lessons learned from 
administrators, nursing staff, and project leaders. Worldviews Evid Based Nurs 2007;4:210-9.

25 Janssen-Heijnen MLG, Maas HAAM, van de Schans SAM, et al. Chemotherapy in elderly small-cell lung cancer patients: Yes we can, but should we do it? Ann Oncol 2011;22:821-6.

26 Tervonen HE, Daniels B, Tang M, et al. Patterns of endocrine therapy in a national cohort of early stage HER2-positive breast cancer patients. Pharmacoepidemiol Drug Saf 2019;28:812-20.

27 Craft PS, Buckingham JM, Dahlstrom JE, et al. Variation in the management of early breast cancer in rural and metropolitan centres: implications for the organisation of rural cancer services. Breast 2010;19:396-401.

28 Fang P, He W, Gomez DR, et al. Influence of age on guidelineconcordant cancer care for elderly patients in the United States. Int J Radiat Oncol Biol Phys 2017;98:748-57.

29 Bristow RE, Chang J, Ziogas A, et al. Sociodemographic disparities in advanced ovarian cancer survival and adherence to treatment guidelines. Obstet Gynecol 2015;125:833-42.

30 Whop LJ, Bernardes CM, Kondalsamy-Chennakesavan S, et al. Indigenous Australians with non-small cell lung cancer or cervical cancer receive suboptimal treatment. Asia Pac J Clin Oncol 2017;13:e224-31.
31 Hodeib M, Chang J, Liu F, et al. Socioeconomic status as a predictor of adherence to treatment guidelines for early-stage ovarian cancer. Gynecol Oncol 2015;138:121-7.

32 Varey AHR, Madronio CM, Cust AE, et al. Poor adherence to national clinical management guidelines: a population-based, cross-sectional study of the surgical management of melanoma in New South Wales, Australia. Ann Surg Oncol 2017;24:2080-8.

33 Lipworth W, Taylor N, Braithwaite J. Can the theoretical domains framework account for the implementation of clinical quality interventions? BMC Health Serv Res 2013;13:530.

34 Page MJ, McKenzie JE, Bossuyt PM. The PRISMA 2020 statement: an updated guideline for reporting systematic reviews. $B M J$ 2021;372.

35 Moher D, Shamseer L, Clarke M, et al. Preferred reporting items for systematic review and meta-analysis protocols (PRISMA-P) 2015 statement. Syst Rev 2015;4:1.

36 Aromataris E, Munn Z. Chapter 7: Systematic reviews of etiology and risk. In: Joanna Briggs Institute Reviewer's Manual. 269. Adelaide, Australia: The Joanna Briggs Institute, 2017. https:// reviewersmanual.joannabriggs.org

37 Sabaté E. Adherence to long-term therapies: evidence for action. Geneva, Switzerland: World Health Organization, 2003.

38 Higgins JPT, Thompson SG. Quantifying heterogeneity in a metaanalysis. Stat Med 2002;21:1539-58. 